\title{
An approach to family-centered coordinated co-management for individuals with conditions identified through newborn screening
}

\author{
W. Carl Cooley, MD', Alex R. Kemper, MD, MPH², and the Medical Home Workgroup of the National \\ Coordinating Center for the Regional Genetic and Newborn Screening Service Collaboratives
}

The care of individuals with rare heritable conditions, such as those detectable through newborn screening, is an important target for quality improvement. Not only is there great opportunity to improve long-term outcomes, but there are lessons that can be generalized to the care of all children with special health-care needs. To identify an approach to quality improvement for individuals with conditions identified through newborn screening, the National Coordinating Center for the Regional Genetic and Newborn Screening Service Collaboratives convened an expert workgroup to develop strategies based on a family-centered, community-based system of care. These recommendations centered on involving families, the primary care medical home, and specialty care providers as equal partners. Key activities

\section{INTRODUCTION}

Newborn screening is one of the most successful public health programs, leading to dramatic improvements in morbidity and mortality for a wide range of heritable conditions. However, there are significant challenges related to the long-term care of affected individuals, including a limited workforce of specialists, reliance on primary care providers who often have limited knowledge about the conditions and their role in management, poorly developed communication systems across the key stakeholders who provide care, and limited resources for coordination of care. Developing strategies to improve health-care delivery for conditions identified through newborn screening could be used as a model for all children with special health-care needs. Concentrating on this smaller population would allow rapid development of tools that could then be widely disseminated.

The challenges to improving care are significant. The business model of most primary care practices is based on highvolume and low-margin clinical encounters, and most coordination activities are not reimbursed. Furthermore, individual primary care practices are likely to have only a small number of patients with conditions identified through newborn screening. Specialty care providers are usually uncertain about the readiness and willingness of primary care providers to participate in the care and management of complex conditions. However, the expansion in the number of conditions for which newborns are screened has created greater demands on the already limited capacity of metabolic and other specialty clinics. Families are to improve care include explicit care coordination, identification of the location of management, and planned co-management. To implement this model of care, the Regional Collaboratives will develop a clearinghouse of tools, engage in activities to evaluate the effectiveness of interventions to improve co-management, and identify strategies to align incentives for health-care providers and families to work together.

Genet Med 2013:15(3):174-177

Key Words: delivery of health care; health transition; integrated; patient care team; patient-centered care

often surprised to discover the amount of fragmentation in the health-care system.

However, targeting individuals with conditions identified through newborn screening has the advantage of bringing unique resources for quality improvement. For example, the Maternal and Child Health Bureau of the United States Health Resources and Services Administration funds seven Regional Genetic and Newborn Screening Service Collaboratives across the country and a National Coordinating Center to improve health-care delivery.

The Regional Collaboratives have focused on care improvement through the medical home model. However, there has been significant uncertainty about what constitutes the fundamental characteristics of this model and how to design effective strategies to foster the medical home for individuals with conditions identified through newborn screening. In response, in 2010, the National Coordinating Center for the Regional Genetic and Newborn Screening Services Collaboratives convened an expert workgroup of health-care professionals, policy makers, and parents of children with heritable conditions. This report summarizes the approach recommended by this workgroup.

\section{STAKEHOLDER IDENTIFICATION}

The workgroup began by recognizing the role of the key stakeholders in improving care for those identified through newborn screening: families, primary care providers, and specialty care providers. The medical home model is based on the assumption 
that families are full partners in all care decisions, and that these decisions must take into account their preferences, goals, and capacity for planned approaches to treatment. ${ }^{1,2}$ Families have varying capacities for understanding, responding, decision making, and resilience, along with varying cultural traditions regarding illness and health.

Primary care providers are expected to provide a familycentered medical home. The word "home" implies a home base or headquarters for care, as well as a care team with whom a patient and family feel familiar and welcome. The American Academy of Pediatrics defines care in a medical home as accessible, continuous, comprehensive, coordinated, compassionate, family centered, and culturally effective. ${ }^{3}$ In rare circumstances, a specialty clinic or special program may provide a medical home for a period of time. However, to do so, such settings must provide comprehensive, longitudinal, and coordinated care and accept first contact responsibility for all preventive, acute, and chronic condition care. ${ }^{4}$

Optimal diagnosis and management of many heritable conditions require specialized knowledge, technology, and expertise. Specialty care providers who often work together as teams are usually needed to confirm the diagnosis and initiate treatment. Specialty care providers provide access to key technologies, to networks of information and registries of patients with similar conditions, and to research and training facilities capable of advancing and disseminating new knowledge. They can develop, monitor, and adjust treatment protocols, facilitate access to treatment (e.g., special medical diets), provide families with up-to-date information, and track broader outcomes, such as developmental progress. However, specialists are often not prepared to provide comprehensive care, such as routine preventive services (e.g., immunizations) or acute illness management not associated with the particular condition. Therefore, most specialty care providers depend on collaboration with primary care providers to assure the best long-term outcomes for their patients.

\section{CARE COORDINATION}

The central goal of care coordination is to link the key stakeholders to assure effective and high-quality care delivery. Ideally, care coordination should include all systems that support the affected individual, including social service agencies and schools. To function across multiple systems or settings, care coordination functionalities must exist within each setting and maintain effective communication links.

Each stakeholder must be informed about evaluation and treatment plans, transitions in care, and their individual roles and responsibilities and each should contribute to planning decisions and timelines. At times, additional elements will need to be part of the care coordination network, including public health agencies, payers, allied health providers, schools, early intervention programs, mental health agencies, durable medical equipment vendors, and pharmacies.

The basic elements of care coordination include an iterative, dynamic care plan, an explicit mutually understood definition of roles, and the identification of the locus of condition management. Many families also value connecting with other families, including those with children who have the same or similar conditions as theirs. Such connections may be facilitated by the primary care medical home or by specialty care providers.

\section{LOCUS OF MANAGEMENT}

Assigning the locus of management for a chronic condition involves explicitly identifying a lead clinical manager or management team and defining the scope of responsibilities. The ideal locus of management for most children is the primary care medical home. However, for some, particularly those with complex conditions identified through newborn screening, it may be a specialty care clinic or team. The locus of management is not synonymous with the medical home and may shift as the acuity, complexity, or severity of health care needs change over time. For example, the locus of management for a child with cystic fibrosis may reside permanently with a specialty clinic team, whereas the locus of management for a child with congenital hypothyroidism may initially be with a pediatric endocrinologist and move later to a primary care clinician with periodic specialty consultation. In both examples, the medical home remains the primary care setting providing preventive care, immunizations, acute illness management, and coordination with community resources. When the locus of management is with the specialty care provider, the primary care medical home often assumes a co-management role for preventive care, clinical triage of acute illnesses, and general chronic care management.

Decisions about the locus of management must include the direct input of the family. Factors that can influence the decision include their comfort and confidence in specific settings or clinicians, their familiarity with and adjustment to the diagnosis, their ability to advocate and assist with the coordination of care for their child, and the distance of their home from the specialty clinic.

\section{PLANNED CO-MANAGEMENT}

Planned co-management reflects the needed communication, coordination, and shared responsibilities across the stakeholders. It involves a proactive, anticipatory approach to care planning through structures (e.g., care plans), processes (e.g., communication), and active input from each team. In many instances, planned co-management requires dedicated personnel responsible for the continuity of co-management plans (care coordinators). To be effective, planned co-management must have the endorsement and engagement of families.

\section{HEALTH-CARE TRANSITIONS}

For individuals identified through newborn screening, three health-care transitions involve particular risks: from shortterm to long-term follow-up, ${ }^{5}$ from hospitalizations to home and community care, and from pediatric to adult health care. These transitions usually involve a transfer or hand-off of some 
or all of the care from one provider or team to another, which can lead to medical errors and fragmentation of care. ${ }^{6}$ Best outcomes of health-care transitions require effective communication and care coordination associated with replicable and efficient processes that include preparation, planning, implementation, follow-up, and closure.

The transition from short-term to long-term follow-up occurs after screening, identification, diagnosis, and initiation of treatment, during which direct monitoring and coordination by state public health resources assists communication among stakeholders, the specialty clinic team, and particularly the family. Once the child's condition is stabilized and the family settles into a "new normal," the health-care focus moves to preventive care, routine screening, immunizations, decisions about acute illness management, and initiation of ancillary supports and services, such as early intervention. The integration of these with chronic condition management and ongoing specialty care may prove challenging as public health surveillance and assistance often diminishes as individuals enter long-term follow-up.?

Hospitalizations, both planned and unplanned, are common for many individuals with conditions identified through newborn screening. Often, hospitalization occurs in tertiary medical centers that are far from the families' homes. At discharge, the transition to home and community may present additional hazards related to inadequate patient education, incomplete medication reconciliation, changed care plans, and poor coordination of primary care, home health care, and other community support. ${ }^{8}$

Increasing numbers of children identified through newborn screening survive into adulthood. Health care is often disrupted during the transition to young adulthood. ${ }^{9}$ Organized and consistent approaches to preparing adolescents and their families for this crucial health-care transition are rarely implemented. ${ }^{9}$ Adult primary and specialty care providers are often inadequately prepared and sometimes unwilling to accept responsibility for the care of young adults with rare or complex conditions of childhood onset. Few pediatric primary and specialty care settings have explicit policies regarding this important life transition and even fewer adult health-care settings have policies addressing the orientation of new young adult patients.

Each of these transitions represents situations in which coordination of care and communication of information is particularly important. If the primary or specialty care providers use tools to promote the coordination of care (e.g., care planning, explicit role definitions, clear identification of the locus of management, and links to other resources), and if these tools are designed for ease of use by all involved, improved coordination and co-management can occur even between settings that have not been equally invested in quality improvement.

Care plans for coordinated co-management require three key structural elements to inform, coordinate, and track care: a brief portable medical summary, an emergency management plan, and a current agenda of active issues. The portable medical summary provides basic demographic information, relevant medical history, a current medication list, contact information for the family, primary care medical home, specialists, and other health-care professionals, and a list of other resources and organizations that may be involved. The emergency management plan combines information about the child's chronic condition with guidance for what constitutes an emergency and what actions should be taken. The agenda of active issues is a constantly evolving list of the next steps in care and support, who is responsible for each step, when each step is to be completed, and who will confirm completion.

\section{NEXT STEPS}

This report was developed to provide guidance on practical steps for using the medical home model to improve care for individuals with conditions identified through newborn screening. The central theme is the need to proactively focus on care coordination, being clear about the locus of control, and implementing planned co-management, especially during periods of medical transition. The Regional Collaboratives are the natural laboratories in which different approaches to implementation are being tested and improved. Activities include the development of care planning and coordination tools to facilitate co-management, enhancing electronic health records to incorporate care planning and communication between the stakeholders, collecting survey data from primary care and specialist care providers and families regarding preferences, concerns, and needs; and supporting busy primary and specialty care settings in the use of quality improvement methods to enhance their capacities for care coordination and communication. As new models of coordination co-management are implemented, their impact on population health, patient and family experience of care, and the costs of care can be evaluated.

\section{ACKNOWLEDGMENTS}

This study was sponsored by the American College of Medical Genetics and the National Coordinating Center for the Regional Genetics and Newborn Screening Service Collaboratives grant U22-MC03957, a cooperative agreement with the Maternal and Child Health Bureau of the Health Resources and Services Administration. We thank the members of the Medical Home Workgroup of the National Coordinating Center for the Regional Genetic and Newborn Screening Service Collaboratives: W. Carl Cooley, MD (chair), Center for Medical Home Improvement, Concord, NH; Gregory Prazar, MD, Exeter Pediatrics, Exeter, NH; Robert Ostrander, MD, Valley View Family Practice, Rushville, NY; Jana Monaco, Woodbridge, VA; Alex Kemper, MD, Duke Clinical Research Institute, Durham, NC; Jane Turner, MD, Michigan State University, East Lansing, Ml; Sondra Gilbert, Cabinet for Health and Family Services, Owenboro, KY; Tamara Hartsell, ARNP, Weatherford, OK; Laura Pickler, MD, University of Colorado School of Medicine, Aurora, CO; Chuck Norlin, MD, University of Utah Health Sciences Center, Salt Lake City, UT; Brad Thompson, The Hali Project, Amarillo, TX; Matt Hirschfeld, MD, Alaska Native Medical Center, Anchorage, AK; Louise Kido Iwaishi, MD, Kapiolani Medical Center for Women \& Children, Honolulu, HI; Marie Mann, MD, HRSA, Rockville, MD; Barry Thompson, MD, 
and Michael Watson, PhD, both at the NCC/American College of Medical Genetics and Genomics, Bethesda, MD.

Disclaimer: The views expressed in this article are those of the authors and do not necessarily reflect those of the Maternal and Child Health Bureau, Health Resources and Services Administration, or the U.S. Department of Health and Human Services.

\section{DISCLOSURE}

The authors declare no conflict of interest.

\section{REFERENCES}

1. Arango P. Family-centered care. Acad Pediatr 2011;11:97-99.

2. Starfield B, Shi L. The medical home, access to care, and insurance: a review of the evidence. Pediatrics 2004;113:1493-1498.
3. American Academy of Pediatrics Medical Home Program Advisory Committee. 2002 Policy statement: the medical home. Pediatrics 2002;113:1545-1547.

4. Starfield B, Shi L, Macinko J. Contribution of primary care to health systems and health. Milbank Q 2005;83:457-502.

5. Kemper AR, Boyle CA, Aceves J, et al. Long-term follow-up after diagnosis resulting from newborn screening: statement of the US Secretary of Health and Human Services' Advisory Committee on Heritable Disorders and Genetic Diseases in Newborns and Children. Genet Med 2008;10:259-261.

6. Coleman EA, Berenson RA. Lost in transition: challenges and opportunities for improving the quality of transitional care. Ann Intern Med 2004;140:533-536.

7. Coleman EA, Parry C, Chalmers S, Min SJ. The care transitions intervention: results of a randomized controlled trial. Arch Intern Med 2006;166:1822-1828.

8. Lotstein DS, Inkelas M, Hays RD, Halfon N, Brook R. Access to care for youth with special health care needs in the transition to adulthood. J Adolesc Health 2008;43:23-29

9. Cooley WC, Sagerman PJ. Supporting the health care transition from adolescence to adulthood in the medical home. Pediatrics 2011;128:182-200. 